\title{
Les organisations populaires du Rwanda : leur émergence, leur nature et leur évolution
}

Jean Nzisabira

\section{OpenEdition}

1 Journals

Édition électronique

URL : http://journals.openedition.org/apad/3783

DOI : 10.4000/apad.3783

ISSN : 1950-6929

Éditeur

LIT Verlag

Édition imprimée

Date de publication : 1 décembre 1992

\section{Référence électronique}

Jean Nzisabira, «Les organisations populaires du Rwanda : leur émergence, leur nature et leur évolution », Bulletin de l'APAD [En ligne], 4 | 1992, mis en ligne le 26 juin 2008, consulté le 10 décembre 2020. URL : http://journals.openedition.org/apad/3783 ; DOI : https://doi.org/10.4000/apad.3783

Ce document a été généré automatiquement le 10 décembre 2020.

Bulletin de l'APAD 


\title{
Les organisations populaires $\mathrm{du}$ Rwanda : leur émergence, leur nature et leur évolution
}

\author{
Jean Nzisabira
}

Le contexte socio-Politique de cette émergence

1 Les organisations populaires du Rwanda revêtent une particularité unique en Afrique : elles ont émergé au début des années 1960 pour combler un vide brusquement créé dans la société traditionnelle par la révolution socio-politique de 1959.

2 Rappelons à cet égard que l'organisation politique, sociale et économique traditionnelle du Rwanda, à laquelle le système colonial belge s'était superposé depuis la fin de la première guerre mondiale, est un régime féodal suivant lequel les ressources (essentiellement la terre et le bétail bovin) étaient réparties entre grands féodataires presque tous de l'ethnie tutsi, dépendants eux-mêmes d'une monarchie très centralisée. Du sommet jusqu'à la base de la pyramide sociale, le gros bétail et la terre étaient dévolus par le biais d'un enchaînement vertical de contrats perpétuels de servage pastoral (ubuhake) ou de servage foncier (ubugererwa).

3 Des pratiques sociales, qui eussent permis aux habitants du Rwanda de s'organiser en associations horizontales d'entraide économique ou sociale, étaient donc inexistantes pour ne pas dire inadmissibles dans ce système féodal. La marque géographique de cette isolation horizontale est à la fois l'atomisation à l'extrême et la dispersion de l'habitat: le pays n'avait ni villes, ni bourgs, ni villages ; mais seulement des fermettes éparpillées dans une fine mosaïque de micro-exploitations agricoles.

La recomposition de l'échiquier socio-politique préparatoire à l'indépendance du pays a brutalement renversé l'ordre social traditionnel. Une lame révolutionnaire, portée surtout par le PARMEHUTU (Parti du mouvement de l'émancipation hutu ${ }^{1}$ ), a en effet emporté le régime féodal en 1959, proclamé la république en 1961 et obtenu l'indépendance du pays en 1962. Cette révolution a donc fauché toutes les relations verticales traditionnelles. Ainsi, le Rwanda s'est trouvé brutalement dépourvu de toute structure sociale opérationnelle sur laquelle s'appuyer pour échafauder et réaliser des 
actions de développement. Le nouveau système politique s'est alors fixé pour principal objectif de combler cette lacune en misant sur les méthodes du développement communautaire ; le caractère fondamental de cet objectif est clairement affirmé dans le tout premier plan quinquennal du Rwanda indépendant. "Les opérations de modernisation rurale, basées en grande partie avant l'indépendance sur la coercition et l'obligation, ne sauraient se concevoir autrement que reposant entièrement sur la persuasion. Un des buts principaux du gouvernement de la République Rwandaise est de promouvoir dans la société nationale des relations horizontales entre communautés familiales, rapports qui faisaient complètement défaut dans l'ancienne société (...). Dans l'optique politique du gouvernement, cette solidarité des collectivités de base se concrétisera par l'éclosion de groupements à forme coopérative mettant en œuvre la philosophie du développement communautaire. Ces groupements seront une véritable école de démocratie, en même temps que d'exercice des responsabilités par leurs membres, l'instrument humainement intégré à partir duquel sera forgée la promotion économique et sociale du pays tout entier" ${ }^{2}$.

5 En vertu de ce projet de société, le système politique républicain s'efforce depuis lors de promouvoir, ex nihilo, un esprit d'auto-promotion par le biais d'organisations populaires à caractère coopératif. A l'heure actuelle, l'esprit d'auto-promotion comprend d'une part une bonne centaine d'organisations formelles (banques populaires et quelques groupements intercoopératifs régionaux) qui procèdent souvent de projets précis à forte composante exogène et d'autre part une myriade d'organisations informelles (tontines et coopératives locales) qui procèdent essentiellement d'une auto-création endogène. Nous nous efforcerons, dans les lignes qui suivent, de donner un éclairage sur la spécificité de chacun des deux types d'organisations en partant de deux cas précis : les banques populaires et les tontines.

Les organisations formelles : le cas des banques populaires Un antécédent considérable : l'expérience coopérative TRAFIPRO

6 L'émergence et le développement des banques populaires au Rwanda procède d'un projet précis qui bénéficie d'une aide technique et financière appropriée en ce sens que l'aide parvient à concilier, autant que faire se peut, la participation populaire et la contrainte technocratique inhérente au fonctionnement d'un système bancaire. Ce projet spécifique des banques populaires a été lui-même précédé d'un autre projet qui l'a sans 'doute inspiré : le projet coopératif TRAFIPRO (Travail, Fidélité et Progrès) dont nous allons brosser les objectifs et la trajectoire historique.

7 A l'origine en 1950, TRAFIPRO est une coopérative locale et endogène; mais elle a par après été nourrie d'une très grande ambition : essaimer son action sur tout le territoire du Rwanda. Puis, suite à une série d'interventions extérieures, son profil originel a été bouleversé de telle sorte qu'elle a perdu sa vocation coopérative.

8 L'aventure a commencé à Byimana ( $15 \mathrm{~km}$ au Sud de Kabgayi) en 1950 par une association d'une dizaine d'instituteurs déterminés à subvenir à leurs besoins de consommation par une série d'initiatives coopératives car, à cette époque, les produits industriels de consommation de masse étaient mal distribués en milieu rural et les commerçants (tous étrangers : européens pour le gros, arabes et pakistanais pour le détail) trop capricieux dans la fixation des prix. En 1951, la petite association ouvre une cantine : restaurant, buvette et quelques autres activités récréatives.

9 Le Révérend Louis Pien, missionnaire catholique des Pères Blancs en poste à Kabgayi, se persuada vite que moyennant un appui méthodologique en matière d'entreprenariat 
coopératif, l'initiative des instituteurs de Byimana pouvait s'ériger en une coopérative plus ambitieuse et faire tache d'huile, notamment via le corps enseignant disséminé partout dans le pays, pour transfigurer à terme le paysage de la distribution et du commerce des produits de première nécessité. Son action pédagogique en ce sens débuta en 1955 ; elle aboutit à la création, le 16 décembre 1956, de la coopérative TRAFIPRO qui obtint la personnalité juridique le 5 février 1957.

10 A son agrément, l'entreprise coopérative TRAFIPRO comptait 140 membres et un capital de 100.000 francs ${ }^{3}$ répartis en deux magasins: un à Byimana, un autre à Kabgayi. Et d'après la structure de ses objectifs, elle devait parvenir à importer régulièrement des produits industriels de consommation populaire en ne recourant qu'exclusivement (ou presque) aux ressources humaines et financières locales afin de démocratiser au maximum l'accès à ces produits. Elle devait en outre assurer la collecte et l'exportation du café pour donner un coup de pouce aux revenus paysans, mobiliser l'épargne et distribuer du crédit populaire, soutenir enfin les efforts tendant à organiser l'économie du pays sous la forme coopérative.

Le projet TRAFIPRO bénéficia, avec bonheur, de la permissivité de la hiérarchie ecclésiastique car l'Evêque de Kabgayi, non seulement céda un terrain sur le domaine diocésain pour accueillir le siège social de la Centrale TRAFIPRO en 1957, mais délia aussi le Père Pien de toute autre responsabilité pour que celui-ci puisse se consacrer pleinement au projet. Le projet TRAFIPRO a créé en outre une formidable synergie avec le mouvement d'émancipation démocratique car les grands ténors du PARMEHUTU, comme Grégoire Kayibanda et Balthazar Bicamumpaka ont été constamment défenseurs de cette chaîne coopérative et un moment donné membres de son conseil d'administration.

Devant l'hostilité des sociétés d'import-export, l'entreprise TRAFIPRO ne put opérer ni importations ni exportations elle-même jusqu'en 1963. Elle se contenta de distribuer en semi-gros et au détail des marchandises importées par d'autres et de collecter du café parche qu'elle revendait à des torréfacteurs-exportateurs. Ainsi, elle répondait déjà à des besoins réels de la population car, très sollicitée, elle s'est rapidement développée en ouvrant de nombreuses succursales (11 déjà en 1961 comptant 9.414 coopérateurs) et en élargissant considérablement sa gamme d'articles de consommation. Cependant, ses cadres étaient sans formation idoine et sans expérience suffisante pour faire face aux redoutables problèmes posés par sa croissance très rapide. C'est ainsi que dès 1961, ses résultats financiers sont systématiquement déficitaires. Et à la fin de l'année 1963, c'est la catastrophe; le volume des pertes cumulées surpasse le montant du capital et des réserves réunis ${ }^{4}$ : impossibilité donc de s'approvisionner encore en marchandises et surtout de ne pas gruger les coopérateurs qui venaient de livrer leur café parche. Le gouvernement, dont les membres les plus influents (notamment le Président Grégoire Kayibanda) avaient une longue affinité avec elle, vola au secours de l'entreprise TRAFIPRO. Celle-ci fut donc sauvée, par les pouvoirs publics, d'une banqueroute certaine ; elle perdit en contrepartie l'essentiel de sa vocation coopérative, notamment l'appropriation et le contrôle démocratique des activités par son sociétariat populaire.

Une brochure diffusée en 1982 par la Direction de l'entreprise ${ }^{5}$ permet de distinguer trois phases cruciales d'intervention des pouvoirs publics dans les affaires de cette coopérative. La première phase date de 1963 lorsque le gouvernement suisse accorde au gouvernement rwandais une aide technique et financière importante destinée à assainir la coopérative TRAFIPRO; la situation financière et la rentabilité de celle-ci 
s'en sont rapidement trouvées améliorées, ce qui a permis un déploiement rapide de l'entreprise (27 succursales) dans les 10 préfectures du pays. La deuxième phase vient en 1966 en réponse à une nouvelle crise provoquée, cette fois-ci, par la dévaluation du franc rwandais, par des malversations financières surtout dans la campagne-café et par de nombreux vols, de sorte que quatre succursales particulièrement touchées ont dû fermer en 1967. De nouveaux crédits sont accordés par le gouvernement suisse; à nouveau la santé financière de TRAFIPRO se normalise et sur la requête de l'Assemblée générale, une politique de remise des responsabilités aux cadres rwandais est entreprise. La troisième phase fait suite aux événements politiques de février 1973, troubles caractérisés surtout par l'expulsion d'employés tutsi des services publics et d'entreprises parastatales. La marche de TRAFIPRO en fut profondément perturbée : une partie du personnel congédié, 15 succursales fermées et la plupart des experts suisses prématurément rentrés chez eux. Après le coup d'Etat militaire du 5 juillet 1973 qui ramena le calme dans le pays, le gouvernement de la deuxième république décide de participer à $90 \%$ au capital social et de dominer ainsi le Conseil d'administration de TRAFIPRO. Cette domination conduira au délestage effectif de la vocation coopérative de TRAFIPRO au profit de celle d'une régie de service public, qui est elle-même en passe d'être privatisée dans le cadre du Programme d'ajustement structurel recommandé par le Fond monétaire international en 1990.

14 En définitive, l'échec de TRAFIPRO en matière de coopérative procède d'un objet social et d'un rythme d'exécution excessivement ambitieux par rapport aux ressources réellement mobilisables, surtout les ressources humaines. Le projet des banques populaires tient compte de cette contrainte ; nous le verrons surtout dans son schéma d'expansion.

L'émergence et le contenu du projet des banques populaires

L'émergence de ce projet se profila publiquement lorsqu'en 1965 le Président Grégoire Kayibanda visita en Suisse le siège de l'Union des Caisses Raiffeisen et se fit expliquer sur place le fonctionnement de celles-ci. Il acquit alors la conviction que ce modèle de mobilisation d'épargne populaire et de distribution du crédit pouvait fonctionner au Rwanda. Il introduisit par la suite une demande d'étude de faisabilité ; la Suisse conclut qu'il fallait tenter l'expérience et accepta en 1972 d'assurer le sponsoring du projet. Des stages de formation furent d'abord proposés à des jeunes Rwandais ; puis, en 1974, un noyau initial de pilotage du projet est installé à Kigali ; c'est ce noyau qui deviendra, en 1986, l'Union des banques populaires du Rwanda. L'architecture du projet, pressentie d'emblée et progressivement mise en place, comprend trois niveaux: un réseau de banques populaires locales liées entre elles, par le biais d'une intercoopération, en fédérations régionales et en Union nationale.

Chacune des banques populaires se caractérise par un territoire de desserte strictement limité à un tout petit territoire car la gestion, essentiellement bénévole, s'appuie sur un très fort sentiment d'identité locale ou, en d'autres termes, sur les liens de proximité entre les membres. Cette restriction spatiale entraine la distinction de deux types d'utilisateurs d'une banque populaire: les sociétaires ordinaires (sociétaires de pleins droits) et les sociétaires affiliés. Pour être sociétaire ordinaire, l'on doit impérativement être résident du territoire de desserte ordinaire, avoir plus de 18 ans et souscrire à une ou plusieurs parts sociales - rémunérée(s) à 5\% - ; aussi, un membre ordinaire peut déposer à titre personnel un montant illimité d'épargne - rémunéré à $3 \%$ - dans sa banque populaire. En revanche, peut être sociétaire affilié, toute personne 
(physique ou morale, sans restriction d'âge) résidant en dehors du territoire de desserte ordinaire qui accepte de souscrire à une et une seule part sociale rémunérée également à $5 \%$. Avec ce statut de sociétaire affilié, l'on ne peut jouir que du seul service de dépôt d'épargne (avec intérêt de $3 \%$ comme les autres) et de retrait, sans accès donc au crédit.

Les fonctions d'administration et de surveillance sont bénévoles; seuls le gérant et le gardien sont rémunérés. Enfin, en dehors des rémunérations des parts sociales et des dépôts, la prise de dividendes est prohibée. Les excédents sont versés à la réserve de fonds propre ou dévolus aux actions sociales de formation des coopérateurs et de diffusion de l'idéologie coopérative.

\section{L'expansion et le financement du projet}

\section{Le schéma d'expansion spatiale adopté par les banques populaires est d'une très rude} exigence: viser prioritairement le Rwanda profond, difficile d'accès; en d'autres termes, ne pas céder à la facilité qui consisterait à tisser le réseau pionnier juste aux alentours des villes notamment de la capitale. L'objectif consistait donc à réaliser assez vite un semis lâche mais équitable de banques populaires locales à partir duquel le mouvement s'étendrait, de proche en proche, à toutes les communautés locales du pays. Le choix de ces implantations pionnières dépendait lui-même d'une série de facteurs: taille des groupements demandeurs, insistance manifestée par leurs porte-parole, gage de sérieux comme par exemple la construction préalable d'un local adéquat destiné à abriter la banque, etc.

Dès 1975, année du lancement effectif du projet, nombreuses sont les demandes d'ouverture de banque qui affluent sans cesse au noyau de pilotage du projet. Elles émanent de bourgmestres, de responsables religieux ou d'autres notabilités locales. L'expansion cumulée du projet se présente comme suit : 13 banques locales comptant 2.016 sociétaires ordinaires en fin 1975,57 banques locales comptant 24.522 sociétaires ordinaires en fin 1980, 79 banques locales comptant 99.585 sociétaires ordinaires en fin 1985 et 119 banques locales comptant 296.026 sociétaires en fin 1990. L'objectif récemment actualisé est d'avoir avant 1995 au moins une banque pour chacune des 145 communes du pays tout en respectant la rentabilité. Si l'on en juge donc par cette expansion, le projet a rencontré beaucoup de succès dans la paysannerie. L'on pourrait même parler de trop de succès car toutes les demandes n'ont pu être satisfaites. Il a fallu réguler drastiquement l'expansion en fonction des tâches incontournables de maturation des groupements demandeurs et de formation du personnel pour ne pas réitérer les erreurs commises antérieurement dans le projet TRAFIPRO. Et devant l'afflux incessant de demandes qu'il s'avoue incapable de satisfaire dans des délais raisonnables, le noyau de pilotage du projet encourage depuis 1986 certains groupements demandeurs à s'ériger eux-mêmes en "Banques ou Caisses de solidarité" autonomes mais susceptibles d'intégrer, à terme, le réseau des banques populaires.

Pour sa réalisation, le projet des banques populaires est surtout tributaire de subventions fournies par la coopération suisse. Dès son ouverture en effet, chaque banque locale bénéficie d'une couverture diachroniquement dégressive de ses frais de fonctionnement : $100 \%$ la première année, $80 \%$ la seconde, $60 \%$ la troisième, $40 \%$ la quatrième, $20 \%$ la cinquième et $0 \%$ à partir de la sixième. Si donc au bout de 5 ans d'exercice la banque locale n'a pas réussi à équilibrer ses comptes, l'appui financier de la coopération suisse se poursuivra sans doute jusqu'à ce que le bilan entre les produits et les charges soit bénéficiaire ou équilibré de manière irréversible. 
Les réalisations socio-économiques du projet

21 utilisé jusqu'à présent. Outre sa simplicité tant pour les déposants que pour les gérants, le livret de compte courant offre aussi l'avantage très apprécié d'une disponibilité permanente du dépôt car l'épargne populaire est essentiellement une épargne de précaution, pour constituer une petite réserve en cas de besoin, ou une épargne de consommation différée en vue d'une dépense ultérieure. Le déposant ne recherche donc pas le rendement maximum, mais plutôt la possibilité de mettre en sécurité son argent et de pouvoir en disposer quand il le veut. Dans cette perspective, le succès des banques populaires a été fulgurant : 13 banques, 2.200 déposants et 13 millions de FRW déposés à la fin de l'année 1975 ; en fin 1990, la situation indique 119 banques, 298.961 déposants et 3.149 millions de FRW déposés.

S'agissant du crédit, la chaîne des banques populaires se dit en faveur de petits crédits au profit du plus grand nombre possible de ses membres au lieu de diriger des sommes énormes vers quelques individus. Le moyen de cet objectif est la décentralisation effective du système qui donne à la caisse locale, à la caisse régionale et à la caisse nationale des compétences bien distinctes. Au niveau des banques locales, les demandes de crédit sont examinées et les décisions prises localement du moins jusqu'à certain plafond qui est actuellement d'un demi-million de RFW par crédit. Au-delà, le crédit est alors du ressort de la caisse régionale ou de la caisse centrale.

En dépit de ce processus de décision démocratique et décentralisé, les faits en matière de crédit sont significativement éloignés de l'équité souhaitée. La confrontation, annuellement réalisée dans les rapports des Banques populaires, entre l'origine de l'épargne et la destination du crédit laisse apparaître systématiquement un transfert important de l'épargne paysanne vers des opérateurs économiques non paysans, surtout les commerçants et les cadres administratifs locaux. Il convient de préciser cependant que ce mouvement s'est quelque peu atténué au cours des années 1980 car, en 1981, la contribution des agriculteurs à l'épargne fut de 50\% mais ils n'ont absorbé en retour que $12 \%$ du crédit distribué, alors qu'en 1990, les mêmes paramètres sont respectivement de $43 \%$ et de $23 \%$. C'est la procédure classique, selon laquelle la banque attend et traite les requêtes spontanées, qui a écarté du crédit mutuelles coopérateurs paysans. D'une part, le paysan est totalement inexpérimenté en matière de formulation de projet bancable: s'équiper, améliorer ou diversifier son activité en utilisant un volume de liquidités largement supérieur à ses disponibilités propres ne relève guère de ses traditions. D'autre part, le crédit n'est octroyé que si le demandeur offre des garanties tangibles, c'est à dire s'il gage des avoirs concrets, des biens réalisables pour permettre au banquier de recouvrer le solde en cas de défaillance. Or en matière de garantie, le paysan est singulièrement démuni. Les terres agricoles, seule valeur paysanne réellement importante et permanente, se prête mal à l'hypothèque car en matière foncière, le Rwanda vit sur un formidable malentendu. D'un côté les ménages agricoles se croient propriétaires des terres qu'ils exploitent et la coutume veut que celles-ci soient transmises intégralement aux descendants mâles. D'un autre côté, l'Etat se considère aussi propriétaire des mêmes terres si bien que celui-ci peut, pour toute cause déclarée d'utilité publique, exproprier un paysan de sa terre sans indemnisation 
foncière. L'indemnisation accordée jusqu'ici dans pareille situation ne concerne que le bâti et les arbres.

Depuis 1985, les responsables des banques populaires sont activement à la recherche de formules de crédit adaptées aux besoins de la paysannerie et à ses capacités de remboursement. Quelques-unes (crédit-soudure, crédit-semences sélectionnées, crédit-bétail, crédit-terrasses...) ont d'ores et déjà été expérimentées, mais elles n'ont pu donner le coup de fouet décisif à l'investissement productif en milieu rural; le problème du crédit y reste donc jusqu'ici sans réponse satisfaisante.

Les organisations informelles : le cas des tontines Spécificité des tontines par rapport aux coopératives locales

Le Rwanda possède deux sortes d'organisations populaires informelles: les coopératives locales (3.168 unités comptant 164.283 membres en 1985) et les tontines (9.243 unités comptant 174.619 membres en 1987). Trois critères permettent de les distinguer aisément.

Contrairement aux coopératives qui ont chacune un patrimoine collectif, les tontines sont généralement des sociétés sans capital social ni biens en gestion commune. Leur objet social et leur fonctionnement sont précisés plus loin.

28 A la différence aussi des coopératives qui sont au moins immatriculées à l'administration communale en attendant d'acquérir pleinement leur personnalité juridique ${ }^{6}$ et qui fonctionnent déjà sur une base contractuelle écrite, les tontines sont encore plus informelles: pas de siège social et les contrats entre membres sont rarement écrits ou en tous cas peu connus de l'autorité administrative. A force cependant d'entendre un discours politique favorable à un épanouissement d'organisations paysannes, les tontines suivent l'exemple des coopératives et se font peu à peu connaître de l'autorité administrative. Mais le facteur déterminant dans cette démarche est d'une toute autre nature; l'enregistrement répond principalement au souci des membres de la tontine de pouvoir recourir ultérieurement aux autorités communales en cas de différends entre eux. Ils recherchent donc une sorte de reconnaissance officielle pour pouvoir bénéficier plus aisément de l'arbitrage de l'autorité en cas de conflits. Le fait de se faire enregistrer spontanément à la commune contre paiement d'une taxe annuelle de 400 FRW montre en effet que les autorités administratives sont de plus en plus perçues comme incontournables dans le règlement des litiges. En 1987, 34\% des tontines en activité sont immatriculées à l'administration communale.

Par opposition enfin aux coopératives, les tontines sont entièrement d'auto-réalisation populaire : pas d'appui technique et financier extérieur comme c'est assez souvent le cas dans des coopératives ; $33 \%$ de celles-ci ont été en effet suscitées par des structures extérieures (l'administration communale, une autorité religieuse, une ONG, un projet de développement rural) et $22 \%$ ont bénéficié d'une libéralité extérieure (un terrain agricole, un moulin, un silo à grain, un don financier).

Objet et fonctionnement des tontines

L'analyse de leur activité permet de classer les tontines rwandaises selon cinq profils bien distincts: tontines financières, tontines d'entraide rotative en travail agricole, tontines d'entraide rotative en travail de construction-logement, tontines d'entraide de type prévoyance mutualiste et tontines en mutation vers l'entraide coopérative. Il convient de souligner cependant que les tontines rwandaises ne sont pas toutes 
monolithiques; un tiers d'entre elles combinent deux ou trois des cinq activités précitées.

Les tontines financières

31 L'entraide rotative en argent entre membres associés est le modèle de tontine le plus courant au Rwanda. Il forme à l'état pur 5.087 cas, soit 55\% et à l'état composite (avec activités d'échange de biens et de services autres que l'argent) 2.897 cas, soit $31 \%$ de tout le mouvement tontinier en 1987.

En principe, c'est un système spécial d'épargne et de crédit conçu pour résoudre à tour de rôle divers problèmes personnels des membres qui forment la tontine. En pratique, c'est une association d'entraide dont le rôle est de collecter régulièrement (semaine, quinzaine, mois, trimestre ou autre) une partie des revenus monétaires d'un certain nombre d'individus qui, chacun à leur tour, peuvent utiliser à leur profit personnel la masse d'espèces ainsi réunie. Les sommes ainsi perçues sont donc affectées à divers projets économiques ou sociaux à la discrétion totale des bénéficiaires. Cependant, il est déjà passé dans les mœurs de ceux-ci $(72 \%$ des cas) la pratique d'informer au préalable leurs associés des projets personnels qu'ils comptent réaliser avec leur tour de tontine. Le respect des engagements pris en tontine exige sûrement une ferme cohésion et une confiance mutuelle au sein du groupe; cohésion et confiance sous-tendues souvent par l'appartenance à un même milieu socioprofessionnel, de manière telle que la solvabilité et la bonne foi de chacun soient assez bien connues des autres membres.

Pour revenir quelque peu au niveau du principe, il est excessivement important de remarquer que le système tontinier d'épargne-crédit ignore superbement, dans le cas du Rwanda tout au moins, la friction temporelle de l'argent ; les sommes épargnées ne produisent pas d'intérêt financier pour l'épargnant. Si donc les dates de collecte de l'épargne sont fort espacées, et si le nombre de membres est important, et partant le cycle complet de la tontine considérable, alors l'inflation peut, à elle seule, étioler la tontine. C'est sans doute pour cela que le sociétariat des tontines financières est généralement restreint (12 membres) et leur cycle court (12 mois).

Quant à leur organisation fonctionnelle, "les tontines d'argent sont toujours les mieux structurées. Les paysans ont donc compris qu'en matière d'argent, on ne badine pas. La seule confiance, dans la gestion, n'est point de mise. Les paysans ont d'ailleurs un adage disant que l'argent a englouti l'amitié. Ils se méfient donc de cette nouvelle puissance qu'est l'argent et s'entourent de toutes les garanties dont ils disposent pour ménager scrupuleusement ses exigences" ${ }^{7}$.C'est ainsi que le fonctionnement des tontines financières s'articule sur un comité de direction composé d'un président, d'un vice-président, d'un secrétaire, d'un trésorier, parfois même d'un "policier" chargé de la discipline au cours des réunions. Le comité de direction doit procéder, à échéance fixe, dans le calme et dans la transparence, à la collecte des mises individuelles et à la remise du résultat au bénéficiaire du tour. L'ordre" des bénéficiaires est établi en début de cycle généralement par tirage au sort et consigné dans un cahier de référence gardé par le président de la tontine. Il faut remarquer cependant que, dans certains cas, deux membres peuvent interchanger leurs tours et demander que leur arrangement soit enregistré dans le cahier de référence. Ceci témoigne bien du caractère flexible des règlements intérieurs des tontines.

En dehors du cahier des tours et des versements, les tontines sont généralement encore dépourvues de règlement écrit. Leur système n'en est pas moins contractuel; la 
régularité des versements des montants convenus est rigoureusement surveillée par tous. Dans la majorité des cas, les retards des versements sont sanctionnés d'une amende (de l'ordre de 10 à $30 \%$ ) qui alimente une caisse commune pour constituer la réserve de fonds dont il a été mention plus haut et/ou pour financer l'ambiance festive des réunions. Dans d'autres cas, les retards justifiés bénéficient d'un moratoire, tout comme les versements anticipés sont autorisés. Nous citerons à cet égard le cas d'une tontine de Kibuye relaté par Jean-Marie Vianney Musabimana et Klaas Tjoelker ${ }^{8}$. Dans cette tontine, il y a effectivement possibilité de payer avec retard ou de payer en avance. Pour quiconque est acculé à payer avec retard, une date de paiement différé est fixée. A cette date, le retardataire va trouver le bénéficiaire de sa cotisation et, ensemble, ils se rendent chez le président pour faire enregistrer le paiement. Si le retardataire dépasse ce délai de grâce, le président le défère devant le tribunal de canton qui évaluera le préjudice à l'aide du cahier des versements. Pour quiconque désire effectuer un paiement anticipé, par exemple pour ne pas courir le risque de garder longtemps sur soi une somme relativement importante, il peut remettre directement, sans autre formalité prescrite par la tontine, le montant au bénéficiaire du tour suivant. Ce bénéficiaire devra alors annoncer en réunion qu'il a déjà touché la cotisation de cette personne. Il faut préciser cependant que dans ces conditions les paiements anticipés se font, logiquement, entre membres de très haute estime réciproque car si le bénéficiaire venait à renier l'encaissement, la tontine serait incapable de régler le différend avec pertinence. Grâce à cet exemple, l'on réalise une fois de plus à quel point les règlements des tontines sont à la fois souples et exigeants. Et comme les tontines foisonnent, sûrement à la satisfaction des membres, l'on peut conclure comme Guy Bédard ${ }^{9}$ que "le caractère informel de la tontine n'enlève rien à ses règles strictes de cycles, de tours, de montants, de procédures".

Concernant la reconduction du contrat et partant la durée de vie d'une tontine, Jean-Marie Vianney Musabimana et Klaas Tjoelker ${ }^{10}$ estiment que les tontines financières ont une certaine continuité car après un cycle, un autre commence mais parfois avec de nouveaux membres. De telles substitutions interviennent lorsque la plupart des membres demandent pour le nouveau cycle d'engager une mise plus élevée; ceux qui s'estiment incapables de tenir le coup quittent alors la tontine pour intégrer ou créer une autre à la mesure de leurs moyens.

En marge de ce modèle aujourd'hui très répandu d'épargne-crédit directement effectué en espèces, il convient de citer deux variantes dont l'épargne (première variante) ou alors la jouissance finale de l'épargne (deuxième variante) n'est pas faite directement en espèces par l'épargnant.

- Dans la première variante, l'épargne-crédit se fait par le biais d'une cotisation en nature notamment la banane (43 tontines en 1987) et parfois aussi d'autres denrées agricoles comme le haricot ou le sorgho (3 tontines). La mise par tour est un nombre préétabli (en général 1) de régimes de banane par membre. Les associés déposent, font mûrir et brassent ensemble ces bananes chez le bénéficiaire du tour. Le vin ainsi obtenu (défalqué cependant de la quantité jugée nécessaire pour se mettre en ambiance de fête) est vendu collectivement, puis le fruit de la vente laissé au propriétaire du tour. En ce qui concerne la transaction en haricot ou en sorgho, la quantité réunie n'est pas vendue collectivement, le propriétaire du tour dispose de ce haricot ou de ce sorgho à sa propre convenance.

- Dans la deuxième variante, les membres de la tontine se stimulent mutuellement à épargner moyennant cotisations régulières en espèces dans le but de pouvoir disposer dans une caisse 
commune et à un moment opportun, soit de quoi payer les frais l'impôt de capitation (9

tontines), soit de quoi payer les frais scolaires des enfants (1 tontine), soit de quoi

renouveler le matériel agricole (2 tontines).

Les tontines d'entraide rotative en travail agricole et en travail de construction-logement

L'entraide rotative en travail agricole ou de construction est quantitativement le deuxième modèle de tontine qui existe au Rwanda. A l'état pur, il ne compte que $8 \% \mathrm{du}$ mouvement tontinier; donc loin derrière l'entraide rotative en argent. Mais à l'état composite, c'est-à-dire en amalgame avec l'entraide rotative en argent ou avec l'exercice de divers secours mutuels, il représente $32 \%$.

En principe et dans la pratique, les tontines de travail fonctionnent suivant le même schéma rotatif d'épargne-crédit, exigeant même cohésion et même confiance entre les membres que les tontines financières. Cependant, le comité de direction pour conduire les opérations et arbitrer les litiges est inexistant ; la seule confiance entre les membres suffit, du moins dans les tontines de travail pures. Chaque membre bénéficie donc, à tour de rôle, du travail de tous les autres. Ceci permet à l'impétrant de liquider en une fois une tâche qui autrement lui coûterait cher en rétribution de main-d'oeuvre ou qui s'étendrait considérablement dans le temps s'il avait à l'exécuter tout seul.

En matière de construction, l'avantage est évident, puisque l'impétrant dispose en un temps record du logement (ou de l'amélioration du logement) souhaité(e), cependant que les autres non encore servis attendent patiemment leur tour. En matière agricole par contre, l'intérêt objectif de la transaction est difficile à percevoir car le besoin d'un supplément de main-d'œuvre est ressenti par tous les ménages agricoles au même moment: les périodes de labour et de semailles. La saisonnalité des gros travaux agricoles doit donc limiter drastiquement chez les membres la patience d'attendre leur tour. La parade à cette restriction est la réduction du cycle à un laps de temps très court (15 à 30 jours) à l'intérieur d'une même saison agricole. Et cette réduction extrême du cycle n'est possible qu'en limitant la taille de la tontine à très peu de membres et en offrant à chacun une ou deux journées seulement de travail collectif. Les enquêtes menées à ce sujet ont montré que les tontines agricoles confinent leur cycle d'activité à l'intérieur d'une saison agricole.

En définitive, une tontine de travail agricole est beaucoup plus subtile que sa consœur de construction-logement car son intérêt majeur est plus psychologique qu'économique. Les membres d'une telle tontine sont persuadés en effet, comme le souligne Jean-Marie Vianney Musabimana ${ }^{11}$, que cultiver en commun fait avancer plus vite les travaux des champs. Mais en réalité, rien ne permet a priori de soutenir que le rendement moyen d'un travailleur en tontine au cours d'une saison agricole est supérieur à celui d'un travailleur hors tontine. Si l'on admet donc que le rendement moyen d'un travailleur en tontine est égal à celui d'un travailleur hors tontine, c'est alors du côté des rapports sociaux entre membres qu'il faut rechercher l'avantage décisif de la transition de travail agricole. D'une part un membre malade, même s'il n'arrive pas à fournir de remplaçant pour maintenir à niveau le volume de main-d'œuvre, continue de bénéficier normalement en son absence du travail de tous les autres membres aux tours qui lui sont prescrits. Sous cet angle, la tontine agricole apparaît donc comme une très judicieuse assurance-maladie pour les agriculteurs. D'autre part, dans le contexte séculaire d'un habitat rural dispersé et d'un système de production pour l'auto-consommation, les ménages agricoles sont coincés dans 
l'isolement, "enterrés" chacun chez eux, sans échange d'idées, sans information. En plus donc du travail accompli, ces prestations de groupe sont des occasions saines d'échange d'idées et de critique d'actualités tous azimuts (événements politiques, faits divers, ...) cela avec humour et bonne humeur, sans le moindre risque de ces désagréables débordements parfois observés dans les rencontres de beuverie.

Les tontines de prévoyance mutualiste

Les clubs d'entraide mutualiste constituent le troisième type de tontine rwandaise en importance numérique : 247 unités, soit $3 \%$ de l'ensemble des tontines recensées en 1987.

D'une manière générale, les tontines de prévoyance mutuelle travaillent sans objet rigoureusement délimité : les interventions improvisées sont légion et leur efficacité en pâtit. En plus, il faut reconnaître que leurs domaines d'activité font également l'objet d'interventions de la solidarité coutumière fondée sur la consanguinité, l'alliance ou le voisinage. Autant dire que la contribution spécifique du mouvement mutualiste est encore extrêmement limitée. Dans 32 tontines, c'est pour parer aux dépenses urgentes en cas d'événements malheureux comme un décès ou un incendie que les gens se sont mobilisés. Dans 215 autres, les membres se sont mobilisés pour assurer le transport de leurs malades : achat d'un équipement collectif (civière notamment) pour le transport pédestre sur courtes distances, participation spontanée à ce transport et/ou petites cotisations pour un fonds de location de véhicule automobile lorsque la spécialisation des soins nécessaires, exige un long déplacement du patient. Il faut signaler aussi que 7 parmi elles ajoutent à ce service de transport une aide supplémentaire à leurs malades, notamment l'achat d'un certain volume de médicaments.

Les tontines en transition coopérative

Les groupements d'entraide de type coopératif forment le dernier type de tontine au Rwanda. C'est en fait un état intermédiaire, un stade transitoire entre tontine et coopérative. Le recensement en a répertorié deux variétés principales dont l'effectif total est de 141 unités ; soit 2\% de l'ensemble du mouvement tontinier en 1987.

La première variété de "tonti-coopératives" (80 unités) concerne une série d'activités de production de biens en commun par les membres: vannerie, broderie, perlerie, couture, cultures vivrières, boisements, etc. La vente, faite également en commun, génère un patrimoine collectif géré plus ou moins démocratiquement à l'image des coopératives locales. Ces tonti-coopératives peuvent évoluer en coopératives en se conformant à la loi: élaboration de statuts écrits, immatriculation au service communal chargé des coopératives...

La deuxième variété, qui compte 61 tonti-coopératives, a trait à l'activité d'épargne et de crédit. Pour près de la moitié d'entre elles, les fonds épargnés sont non seulement faibles, mais aussi constamment détenus au domicile de l'un des membres avec tous les dangers de vols. Ils sont alimentés par les fameuses amendes perçues auprès des retardataires en tontines financières ou par de petites sommes versées à titre d'épargne individuelle. De petites sommes en sont souvent prélevées pour être accordées à titre de prêt (généralement sans intérêt) à des membres confrontés à des situations d'urgence (maladie, décès,...). Dans l'autre moitié des tonti-coopératives d'épargne et de crédit, les fonds sont relativement importants et surtout systématiquement versés dans une institution bancaire sur un livret d'épargne collectif. Les membres entreprenants peuvent en retirer un service important, proportionnellement à leurs dépôts. En gageant leur solidarité auprès des banques 
populaires, ces tonti-coopératives avalisent des crédits individuellement sollicités par leurs membres.

Émergence et diffusion des tontines

47 L'origine des tontines rwandaises est difficile à établir avec précision. De .nombreuses similitudes avec les secours ponctuels traditionnellement pratiqués dans le cadre de la solidarité coutumière font naturellement supposer que les tontines sont le résultat d'un processus de mutation de formes préexistantes vers des structures d'entraide résolument au-dessus du lien parental et cela sous l'effet d'un usage de plus en plus radical de la monnaie. Cette hypothèse de mutation des structures sociales sous l'égide de la monnaie est judicieuse; elle permet d'expliquer sans doute la rapidité avec laquelle les tontines se répandent dans le pays. Mais elle ne répond pas aux questions quand ? Et comment? Le mouvement tontinier a commencé.

Une pratique mutualiste de mouture européenne, aujourd'hui disparue, permet d'expliquer l'émergence d'une petite partie seulement du phénomène tontinier; il s'agit de mutualités chrétiennes créées à Butare et à Nyundo en $1954{ }^{12}$. Le pôle de Nyundo étendait son influence sur l'actuelle préfecture de Gisenyi, de Ruhengeri, de Cyangugu et sur une partie de Kibuye ; tandis que le pôle de Butare s'occupait du reste du pays. L'un et l'autre comptaient donc un grand nombre de sections mutuelles de base et fonctionnaient normalement jusqu'à s'unir, en 1959, en Fédération des mutualités rwandaises (FRW) avec l'aide technique et financière de l'Alliance nationale des mutualités chrétiennes de Belgique. Ces mutualités furent hélas profondément perturbées par les vissicitudes de la révolution politique de 1959. Repris en 1963, leur fonctionnement ne survivra pas longtemps à la mauvaise gestion ainsi qu'aux charges trop lourdes par rapport aux ressources. Leur délisquescence inspira cependant le lancement en août 1975 de la chaîne des tontines mutualistes "umubano mu bantu" dans la préfecture de Butare ${ }^{13}$.

L'essentiel du mouvement tontinier trouve son émergence dans les migrations alternantes de l'époque coloniale entre le Rwanda et les territoires britanniques d'Afrique orientale. Les premières organisations tontinières ont pris naissance dans la périphérie orientale du pays : ibimina (tontines financières) en préfecture de Byumba et muvandimwe (tontines composites d'entraide rotative en argent, de mutualité, ...) en préfecture de Kibungo. Cette localisation périphérique des premières tontines n'est donc pas le fruit d'une génération spontanée car les tontines financières existent depuis bien longtemps en Uganda.

Pour expliquer précisément l'émergence des tontines financières dans la région frontalière de Byumba, la mémoire collective relate l'aventure de deux jeunes gens (Karera et Karuhanyi) qui se sont un jour expatriés en Uganda à la recherche d'un travail salarié. Ils réalisèrent bien vite que leur salaire mensuel couvrait assez largement leurs besoins ordinaires de consommation mais ne permettait à personne de mettre de côté une somme suffisante à envoyer en famille. En adoptant le système d'épargne tontinière qu'ils avaient pu observer chez les salariés ugandais, ils parvinrent à envoyer alternativement, chacun à sa famille, la cagnotte mensuelle de leurs cotisations.

51 En préfecture de Kibungo, les associations muvandimwe ont commencé dans des circonstances analogues. Israël Sefigi, fondateur de muvandimwe en 1966, avait connu auparavant quelques associations d'entraide tontinière en Tanzanie. Selon ses propres propos, Sefigi s'est attelé d'abord à mobiliser son entourage contre une tradition qu'il 
trouvait très fâcheuse: l'irrespect envers les défunts ${ }^{14}$. La tradition consistait à enterrer les défunts vaille que vaille enveloppés dans les feuilles de bananier ou dans des torchons ou paillassons déjà relégués dans les oubliettes de la hutte familiale. Pour Sefigi, il fallait à tout prix infléchir la tradition afin de donner aux défunts des funérailles honorables. Ses campagnes de sensibilisation suscitèrent rapidement de petites .caisses mutuelles alimentés par une cotisation mensuelle de 2 francs rwandais par membre. Sefigi s'est évertué ensuite à étendre cette entraide mutuelle à l'évacuation des malades sur les dispensaires, les centres de santé et les hôpitaux en systématisant l'acquisition d'au moins une civière par groupement muvandimwe. Les frais d'hospitalisation et de médicaments sont même, dans certains groupements, entièrement pris en charge par la mutuelle. Fort de ces deux succès, Sefigi a introduit enfin dans ses mutuelles l'entraide rotative en travail ainsi que l'entraide rotative en argent qui permirent la construction de nombreux logements durables et plus confortables que la paillotte parabolique traditionnelle. Le succès fut tel que muvandimwe s'est structuré, depuis 1978, en trois niveaux qui, en 1986, comptent 190 sections locales, 11 intergroupements à l'échelon communal et une Union des intergroupements au niveau de la préfecture de Kibungo ${ }^{15}$.

Conclusion

Depuis 1960, un mouvement d'associations populaires s'enracine dans la société rwandaise. Il prend hardiment la niche perdue, suite à la révolution de 1959, par les rapports verticaux que chapeautait l'aristocratie pastorale ou terrienne. Ce mouvement, à forte coloration coopérative ou mutualiste, comprend aujourd'hui deux axes : un axe d'associations formelles (surtout les banques populaires) qui procèdent de projets précis à forte composante exogène et un axe d'associations informelles (les tontines par exemple) d'auto-réalisation endogène. L'un et l'autre ont connu une croissance très considérable : $24 \%$ par an pour les tontines et $20 \%$ par an pour les banques populaires entre 1975 et 1985.

Pour autant donc que le régime politique n'y soit pas hostile, un mouvement d'associations informelles peut s'épanouir dans les couches populaires sans la moindre instigation des pouvoirs publics ni de toute autre structure externe. C'est ce qu'indique précisément le succès des tontines au Rwanda. Grâce à son système par lequel les personnes s'obligent par petits groupes à épargner pour jouir tour à tour d'un volume d'argent ou de travail très important, l'activité tontinière permet en effet à plusieurs centaines de milliers d'adhérents d'atteindre un certain nombre d'objectifs autrement irréalisables: acquisition de logements confortables, de bicyclettes, de radio-récepteurs, de terres agricoles, du bétail, de quelques services sociaux divers, etc...

De même, des projets conçus par "le haut", c'est-à-dire par les pouvoirs publics nationaux éventuellement appuyés par une aide extérieure appropriée, peuvent gagner l'adhésion des populations-cible. L'expérience de TRAFIPRO et des banques populaires autorise largement cette affirmation. Cependant, de tels projets n'enracinent durablement leurs acquis que si le rythme de réalisation c9rrespond aux ressources humaines locales réellement mobilisables, que si le rôle de l'Etat reste discret, que si la bureaucratie est limitée au strict nécessaire. 


\section{BIBLIOGRAPHIE}

Bédard, G. (1986), Argent chaud et argent froid : la mobilisation de l'épargne locale par des institutions coopératives et son impact sur le développement local, Cahiers de l'Université coopérative internationale, $\mathrm{n}^{\circ} 7,35 \mathrm{p}$.

Burkhardt (1965), Note sur le mouvement coopératif rwandais, Paris, BDPA, Rapport d'une mission au Rwanda, Opération n ${ }^{\circ}$ 15-06-63,99 p.

Ministère des Affaires Sociales (1962), Plan quinquennal d'assistance au développement rural et d'action coopérative 1963-1967, Kigali, République Rwandaise, 66 p.

Ministère des Finances et de l'Economie (1987), L'économie rwandaise : 25 ans d'efforts (1962-1987), Kigali, République Rwandaise, 233 p.

Mugesera, A. (1987), Aperçu sur la pratique mutualiste au Rwanda de 1954 à 1976, in "Séminaire de concertation sur les organisations mutuelles au Rwanda tenu à Kigali du 10 au 12 mars 1987", Kigali, CFRC-IWACU, pp. 5-11.

Mukaka Yumba, E. (1983), Étude d'un cas rwandais : le muvandimwe. Muvandimwe et développement rural, Actes de l'Université coopérative internationale d'hiver à Yaoundé-Saa, 17-28 janvier 1983, numéro spécial : solidarité traditionnelle et développements mutualistes, pp. 77-80.

Musabimana, J.M.V. (1986), Identification des sections de l'association muvandimwe, Kigali, CFRC-IW ACU, 85 p.

Musabimana, J.M.V. (1990), Inventaire des groupements sans gestion commune 1987, Kigali, CFRC- IW ACU, 53 p.

Musabimana, J.M.V. et Mugesera, A. (1986), Les groupements spontanés en milieu paysan rwandais : synthèse de cas, Kigali, CFRC-IW ACU, 47 p.

Musabimana, J.M.V. et Tjoelker, K. (1985), Les organisations spontanées en milieu paysan : étude menée dans la zone du Projet agricole de Kibuye, Kigali, CFRC-IW ACU, 68 p. + annexes.

Nzisabira, J. (1992), Participation populaire au processus de développement du Rwanda : les idées et les faits, Louvain-la-Neuve, Cahiers du CIDEP, $\mathrm{n}^{\circ} 13,327 \mathrm{p}$.

Trafipro (1982), Coopérative TRAFIPRO (Travail, fidélité, progrès) : 25 ans, Kigali, 24 p.

Willot, P. (1983), Etude d'un cas rwandais : le muvandimwe. Entretien avec un fondateur, Actes de l'Université coopérative internationale d'hiver à Yaoundé-Saa, 17-28 janvier 1983, Numéro spécial : solidarité traditionnelle et développements mutualistes, pp. 74-80.

\section{NOTES}

1.L'ethnie hutu, très largement majoritaire (85\%) était économiquement surexploitée et même socio-politiquement opprimée par le biais des deux institutions susmentionnées au profit d'une petite aristocratie issue de l'ethnie tutsi.

2.Ministère des Affaires sociales (1962), Plan quinquennal d'assistance au développement rural et d'action coopérative 1963-1967, p. 12.

3.Il s'agit du franc congolais devenu franc du Ruanda-Urundi le 22 septembre 1960, puis franc rwandais (FRW) le 14 avril 1964. Ce franc gardera son équivalence avec le franc 
belge jusqu'à la première dévaluation (de 100\%) en février 1966. (cf. Ministère des Finances et de l'Economie (1987), L'économie rwandaise : 25 ans d'efforts, p. 34).

4.Burkhardt (1965), Note sur le mouvement coopératif rwandais, p. 49.

5.TRAFIPRO (1982), Coopérative TRAFIPRO (Travail, Fidélité, Progrès) : 25 ans, 24p.

6.La reconnaissance juridique est pour les coopératives très difficile à obtenir. En 1985, moins de $5 \%$ étaient juridiquement reconnues et rien ne permet de penser que ce pourcentage ait été sensiblement augmenté depuis.

7.Jean-Marie Vianney Musabimana et Antoine Mugesera (1986), op. cit., p. 43.

8.Jean-Marie Vianney Musabimana et Klaas Tjoelker (1985), Les organisations spontanées en milieu paysan : étude menée dans la zone du Projet agricole de Kibuye, EP.21-24. .

9.Guy Bédard (1986), Argent chaud et argent froid : la mobilisation de l'épargne locale par des institutions coopératives et son impact sur le développement local, p. 4.

10.Jean-Marie Vianney Musabimana et Klaas Tjoelker (1985), op. cit., p.55.

11.Jean-Marie Vianney Musabimana (1990), Inventaire des groupements sans gestion commune 1987, p. 7.

12.Cf. Antoine Mugesera (1987), Aperçu sur la pratique mutualiste au Rwanda de 1954 à 1976, pp. 1-11.

13. Communication personnelle du fondateur de la chaîne, Jonas Ndayisaba. En 1990, cette chaîne comptait 15 sections de base. La section de Cyarwa, née en août 1982 avec 153 membres, est la plus dynamique de toutes; elle compte 819 membres en fin 1988.

14.A ce sujet, l'on peut se référer à Paul Willot et à Edith Mukakayumba dont les contributions sont précisées en bibliographie.

15.Jean-Marie Vianney Musabimana (1986), Identification des sections de l'association muvandimwe, p. 3.

\section{AUTEUR}

JEAN NZISABIRA

CIDEP, Louvain-la-Neuve (Belgique) 